\title{
Numerical Simulation and Experimental Analysis of Volume Alternate Cavitation (VAC) - A new Cavitation Generation Method
}

\section{Shangshuang Chen}

Jiangsu University

Yun Wang ( $\nabla$ wangyun@ujs.edu.cn )

Jiangsu University https://orcid.org/0000-0001-6521-0445

Fuzhu Li

Jiangsu University

Shenwei Xue

Jiangsu University

Zhenying Xu

Jiangsu University

Chao Yu

Jiangsu University

Kun Zhang

Jiangsu University

\section{Original Article}

Keywords: cavitation generation method, volumetric alternate cavitation (VAC), generating mechanism, evolution law, computational fluid dynamics (CFD), cavitation flow visualization (CFV)

Posted Date: December 20th, 2021

DOI: https://doi.org/10.21203/rs.3.rs-1143880/v1

License: (c) (1) This work is licensed under a Creative Commons Attribution 4.0 International License. Read Full License 


\section{Abstract}

Cavitation generation methods have been applied in multifarious directions due to their diversity. And scholars have carried out numerous researches and discussions on cavitation generation methods. The purpose of this study is to explore the generating mechanism and evolution law of volumetric alternate cavitation (VAC). In the VAC, the liquid water is placed in an airtight container with variable volume. With the volume alternately changes, the liquid water inside the container continues to cavitate. In this study, the mixture turbulence model and in-cylinder dynamic grid model were used to apply computational fluid dynamics (CFD) simulation of volume alternate cavitation. In the simulation, the cloud images at 7 heights on the central axis are monitored, and the phenomenon and mechanism of height and eccentricity are analyzed detailedly. By using the method of cavitation flow visualization (CFV), the generating mechanism and evolution law of cavitation are clarified. The synergistic effects of experiments and high-speed camera capture confirm the simulation. In the experiment, the volume change stroke of the airtight container is $20 \mathrm{~mm}$, the volume change frequency is $18 \mathrm{~Hz}$, and the shooting frequency of the high-speed camera is set to $10000 \mathrm{fps}$. The results show that the position of occurring cavitation phenomenon has a reasonable law during the whole evolution cycle of the cavitation cloud. It is evident that a cycle of volume alternation corresponds to the generation, development and collapse stages of cavitation bubbles.

\section{Introduction}

Nowadays, cavitation generation method mainly includes hydrodynamic cavitation (1-5), ultrasonic cavitation (6-9) and laser-induced cavitation (10-14), and its application covers cleaning $(15,16)$, surface treatment $(17-22)$, cavitation molding $(23,24)$, environmental protection and renewable $(25,26)$, etc.

The study of the traditional cavitation generation method has been concerned by many researchers. The theory and application of hydrodynamic cavitation, an important cavitation generation method, have been paid much attention and studied by scholars. A systematic and detailed study on hydrodynamic cavitation for disinfection is conducted (2). The energy release of cavitation phenomenon and its mechanism are described, and the effectiveness, economy and applicability of hydrodynamic cavitation disinfection are confirmed. Pooja et al. (1) studied the degradation of benzene in wastewater by hydrodynamic cavitation in combination with air. Through the experimental study of inlet pressure, thermal conductivity, treatment duration and air flow ratio, the optimal parameter setting that can be used to degrade benzene is obtained. Panda (27) et al. made a detailed report on the development trend and practical application of hydrodynamic cavitation in recent years, and pointed out the future development direction in view of specific deficiencies. In addition to hydrodynamic cavitation, many other cavitation generation methods have been studied. The effect of viscosity reduction is studied through the synergistic effect of ultrasonic cavitation and heterogeneous catalyst (8). Shen et al. (9) applied ultrasonic cavitation to medicine and studied the effects of different parameters in ultrasonic cavitation on inducing apoptosis of tumor cells. Abhinav et al. (10) explored the impact force of a single bubble rupture in the case of the combination of laser-induced cavitation and acoustic cavitation. Pello et al. (25) 
reported the combination of ultrasonic cavitation and ozone to treat wastewater, and the treated wastewater met the standards for microbial disinfection of drinking water. Obviously, these traditional cavitation generation methods can be applied to life and production after research.

Although cavitation research has a long history, the research on the mechanism of cavitation generation methods and cavitation evolution law has not interrupted. Sun (28) analyzed the cavitation mechanism and development process of the cavitation flow field located on the rotor and stator, and concluded that the shape and size of the vortex cavitation depended on the compression effect generated by the interaction. A new type of rotary hydrodynamic cavitation generator has been studied and applied to the refining of cellulose pulp, which proves that this method is more economical and effective than conventional methods $(29,30)$. Chen et al. $(31,32)$ designed experimental equipment for cavitation generated by negative pressure, studied the erosion effect of negative pressure cavitation on material removal rate and surface roughness, and proposed a kind of cavitation water-suction polishing without grinding conditions to remove materials through green manufacturing method. Among them, the method used by researchers to capture the cavitation phenomenon is cavitation flow visualization (CFV) $(7,11$, $28,30,33)$. The dynamic evolution law of cavitation cloud of submerged water jet was obtained by visualization of cavitation flow (34). Steve (35) studied the occurrence and evolution of cavitation in capillary tubes under the condition of temperature alternation, and made corresponding theoretical analysis and experimental discussion. In evidence, in order to break through the limitations of traditional cavitation generation methods, novel cavitation generation methods emerge in an endless stream.

In this paper, a new cavitation generation method, volumetric alternating cavitation (VAC), is proposed. Different from the traditional cavitation generation methods, the mechanism of VAC is based on the change in volume of the airtight container to form a low- pressure environment lower than the saturation vapor pressure of the liquid phase, and then the cavitation bubbles are formed in the low-pressure environment, and finally the cavitation bubbles are broken by high pressure extrusion. Due to the volume changes in the airtight container, the cavitation bubbles are constantly generated and broken. However, cavitation bubbles rupture $(14,36)$ will generate extremely huge energy, which can play a role in polishing and strengthening the wall surface, especially for the inner surface of complex cavities. In addition, VAC can also be used for rapid emulsion manufacturing, cavitation cleaning and other aspects. Of course, the advantages of this cavitation generation method are also distinct, such as: the method of VAC is simple and easy to operate, the device of VAC is environmentally friendly and energy saving. Moreover, this study can provide guidance for the application and design of VAC in the future.

\section{Experimental Facility}

As shown in Fig.1, an experimental platform was built to meet the experimental requirements. The experimental platform includes a cavitation generator (f), a shooting device, a motor drive device (b), a control terminal (c) and supports. The cavitation generator ( $f$ ) is an airtight container made of assembled parts, which can meet the requirement that there is only water in the airtight container without excess air 
pressure. The sample (g) in the cavitation generator ( $\mathrm{f}$ ) has a hole $30 \mathrm{~mm}$ deep and $4 \mathrm{~mm}$ in diameter inside. The shooting device is composed of a high-speed camera (d), a high-power LED light (e) and reflective glass. The shadow imaging method (6) is used to capture the phenomenon of the VAC. In order to reflect the complete period of the experimental phenomena in detail, the time interval of the high-speed camera (d) is set to 10,000 FPS. The motor drive device (b) is used to drive the reciprocating movement of the push rod, and then realize the reciprocating movement of the piston inside the cavitation generator (f), to meet the conditions of generating VAC. Fig. 2 is a schematic diagram of the device shown.

Refer to Figure 2, the experimental details can be summarized as follows:

1. The environment for cavitation is an airtight container, which can well avoid the exchange of gas with the outside.

2. The airtight container is a cylinder which consists of three faces: the upper surface, the side surface and the bottom surface. Inside the container sits a cylindrical sample with a small hole.

3. The side and the bottom surface of the airtight container are fixed, and the upper surface is essentially the bottom surface of the piston.

4. The piston has two vital functions, one is the sealing ring around the piston to ensure the airtight container is sealed. The other is that the piston can move up and down under the drive of the motor and linkage to realize the volume alternating of the airtight container.

\section{Numerical Analysis}

\subsection{Theory}

\subsubsection{Mixture model}

The Mixture model in CFD can be used for two-phase or multiphase flow calculation. The model allows interphase penetration, that is, the sum of the volume fraction of each phase in each grid cell can be any value between 0 and 1 . Of course, the model also allows for phase slip, meaning that each phase can have different velocities. Through the analysis, Mixture model is suitable for the numerical analysis of this study. Its momentum conservation equation is: 
$\frac{\partial}{\partial t}\left(\rho_{m} \vec{v}_{m}\right)+\nabla\left(\rho_{m} \vec{v}_{m} \vec{v}_{m}\right)=-\nabla p+\nabla\left[\mu_{m}\left(\nabla \vec{v}_{m}+\right.\right.$

$\left.\left.\nabla \vec{v}_{m}^{T}\right)\right]+\rho_{m} \stackrel{\grave{g}}{+} \vec{F}-\nabla\left(\sum_{k=1}^{n} \alpha_{k} \rho_{k} \vec{v}_{d r, k} \vec{v}_{d r, k}\right)$

Where, $\mathrm{n}$ is the number of phases, $\vec{F}$ is the volume force, and $\mu_{m}$ is the viscosity of the mixed phase:

$\mu_{m}=\sum_{k=1}^{n} \alpha_{k} \mu_{k}$

$\vec{v}_{d r, k}$ is the drift velocity of the quadratic term.

$\vec{v}_{d r, k}=\vec{v}_{k}-\vec{v}_{m}$

\subsubsection{Dynamic mesh model}

Regarding the dynamic mesh, the integral form of the general scalar in the conservation equation is $\Phi$ Under the premise of any controllable volume $V$, the boundary motion of the mesh can be expressed as:

$$
\begin{aligned}
& \frac{d}{d t} \int_{V} \rho \Phi d V+\int_{\partial V} \rho \Phi\left(\vec{u}-\vec{u}_{g}\right) d \vec{A}=\int_{\partial V} \Gamma \nabla \Phi d \vec{A}+ \\
& \int_{V} S_{\Phi} d V
\end{aligned}
$$

Where $\rho$ is the density of the fluid, $\vec{u}$ is the velocity vector, $\vec{u}_{g}$ is the moving velocity of the moving mesh, $\Gamma$ is the diffusion coefficient, and $S_{\Phi}$ is the source term of $\Phi$ Here, $\partial V$ is used to express the boundary of the controlled volume $\mathrm{V}$.

\subsection{Numerical analysis of complete period}

In order to reveal the specific mechanism of VAC, the reciprocating motion of the piston is used to control the alternate change of volume. Therefore, this study takes an alternate change of volume as the basis for simulation. Fig. 3 is the initial simulation model. The height of the inner hole in the sample was equally divided into 6 sections, with a total of 7 heights, and the change of the gas phase volume fraction was monitored at the 7 heights within a motion period. Fig. 4 is the distribution of different heights in the piston inner hole.

\subsubsection{Gas phase volume fraction distribution cloud nephograms}

The volume variation in this study is based on the motion controllability of the upper interface. In the simulation process, the upper interface takes $18 \mathrm{~Hz}$ as the motion frequency and $20 \mathrm{~mm}$ as the motion stroke to monitor the specific situation of the gas phase volume fraction within a cycle and make the 
corresponding simulation cloud map. Fig. 5 shows the cloud nephograms of the gas phase volume fraction at seven heights in the inner hole.

\subsubsection{Influence of height on cavitation formation}

As shown in Fig. 6, this figure demonstrates the data diagram of gas phase volume distribution at different heights along the central axis of the inner hole with time variation. It is obvious that the gas phase volume fraction varies constantly over time at each height.

Wherein, the height of 0 represents the change rule of the gas phase volume fraction at the bottom of the hole. It is found that the gas phase volume fraction at the bottom of the hole is relatively high at the beginning, then decreases continuously. The reason for this phenomenon is that in the initial process, the liquid will be attached to the piston due to the tension, and the upward movement of the piston will drive the liquid upward. There will be a low-pressure area at the bottom of the liquid. When the pressure of the low-pressure area is lower than the saturated vapor pressure of the liquid, the liquid will be transformed into gas. After the initial process, due to gravity, the liquid attached to the piston separates from the piston and begins to deposit downward. The generated gas, which is less dense than the liquid, floats upward through the liquid, resulting in a declining volume fraction of the gas phase at the bottom of the hole.

The height of $0.03 \mathrm{~m}$ represents the situation of the gas phase volume fraction on the upper surface. It can be clearly seen from the figure that the gas phase volume fraction on the upper surface is more than that at other heights of the inner hole. The reason can be summarized as follows: when the piston is moving, the liquid is always inside the hole due to gravity, so there is a low-pressure area like vacuum on the upper surface, as shown in Fig. 7. The pressure in this low-pressure area is much lower than the saturated vapor pressure of the liquid, causing the liquid on the upper surface to undergo a violent phase transition, constantly changing from liquid to gas. The gas phase volume fraction on the upper surface appears at the middle stage and the last stage of the piston movement cycle. The two peak values of gas phase volume fraction appear in the middle stage and the final stage of piston motion cycle respectively. The reason for the peak in the middle stage is that at this time point, when the piston moves to the limit stroke, the liquid phase in the inner hole is transformed into the gas phase by the most amount, and the generated gas is mostly filled in the vacuum-like low pressure area above the liquid. As the generated gas is more, the liquid level will decline, leading to the higher volume fraction of the gas phase on the upper surface. The reason for the peak in the final stage is that the piston begins to return to the initial height. Due to the excessive speed, the gas in the vacuum-like low-pressure area has been pushed back into the hole before being converted into liquid, resulting in a sharp increase in the gas phase volume fraction at this time.

\subsubsection{Influence of eccentricity on cavitation formation}

In view of the above results, this study conducted the exploration on the upper surface and bottom surface of the inner hole, and analyzed the relationship between the gas volume fraction in these two 
planes and the eccentricity (the distance from the central axis of the inner hole). The specific processing results are shown in Fig. 8 and Fig. 9.

Fig. 8 shows the variation of gas phase volume fraction with different eccentricity on the upper surface over time. It can be clearly seen from the figure that the gas phase volume fraction is generally maintained above 0.7 , which belongs to a relatively high level. However, when the eccentricity is $0.002 \mathrm{~m}$ and the time is $0.0034 \mathrm{~s}$, the gas phase volume fraction decreases sharply to less than 0.4 . The reason can be summarized as follows: at 0.0034 s, the piston moves to the limit stage of stroke. At this stage, the velocity of the piston is close to zero. Meanwhile, the conversion rate from liquid phase to gas phase reaches a very low level, and the conversion from gas phase to liquid phase begins. The area with an eccentricity of $0.002 \mathrm{~m}$ belongs to the near wall surface, and the newly generated liquid phase will be preferentially adsorbed to the wall surface, giving rise to a sharp decrease in the volume fraction of the gas phase there.

Fig. 9 shows the variation of gas phase volume fraction with different eccentricity at the bottom surface over time. It can be explicitly seen from the figure that the volume fraction of the gas phase decreases over time, and different eccentricity has little effect on the volume fraction of the gas phase.

\section{Results And Discussion}

\subsection{Formation stage of cavitation bubbles}

In order to verify the correctness of the simulation results, plenty of experiments were carried out, and a high-speed camera was used to capture the phenomenon. Meanwhile, the method of CFV is used flexibly here. Fig. 10 shows the cavitation situation in the hole when the volume just starts to increase. It is apparent from the picture that: As the volume increases, the cavitation bubbles are generated at a height of 0 , and there are a large number of cavitation bubbles on the upper surface, that is, the primary stage of cavitation, which is consistent with the explanation given in Fig. 5 that cavitation occurs preferentially on the bottom and the upper surface. Fig. 11 shows the schematic diagram of initial generation of cavitation bubbles at the bottom. The cavitation generation stage reveals the process of the cavitation bubbles from nothing to existence, which confirms the feasibility of VAC.

\subsection{Development stage of cavitation bubbles}

As the volume continues to increase, the cavitation cloud begins to evolve further. As shown in Fig. 12, the rising of the piston drives the cavitation bubbles near the upper interface to move upward. And the cavitation cloud separation phenomenon appears on account of the bubbles farther away from the upper interface having the tendency of downward movement. The violent cavitation reaction at the upper interface also corresponds to the explanation given in Fig. 5. In the process of separation, the large bubble is torn (35) and new small bubble begins to form.

4.3 Collapse stage of cavitation bubbles 
Cavitation bubble collapse mainly occurs at the end of the volume alternating cycle, that is, the piston returns to the liquid level again. As shown in Fig. 13, when the piston moves to the end of a period, a plentiful of gas phases appear on the upper surface of the liquid, which is consistent with the interpretation of the simulation results. However, cavitation collapse is particularly significant at this stage, with large cavitation bubbles collapsing into a great deal of small cavitation bubbles. Fig. 14 is a schematic diagram of cavitation bubble collapse.

\subsection{Discussion}

In the condition of an ideal gas, we can assume that $\mathrm{P} * \mathrm{~V} / \mathrm{T}$ is constant in an airtight container. Hence, $\mathrm{P}$ is inversely proportional to $\mathrm{V}$ when the temperature constant. That is, the pressure inside the airtight container decreases as the airtight container volume increases. When the volume increases to a certain extent, the pressure $\mathrm{P}$ must be less than the saturated vapor pressure of the liquid. Therefore, the liquid inside the container is going to be converted into a gas. On the other hand, when the volume decreases, the gas turns into a liquid.

In the airtight container, the gas and liquid are cyclically converted, and the corresponding phenomenon is the formation and collapse of cavitation bubbles, such as the cavitation phenomenon photographed by the high-speed camera.

Compared with the traditional method of indirectly generating low pressure by using the velocity difference in water, this study directly creates a low-pressure environment similar to vacuum, which is a completely new cavitation generation method.

\section{Conclusions}

In this study, numerical simulation and experimental analysis of VAC were carried out, and the following conclusions were drawn.

1. VAC is feasible as a new cavitation generation method. The whole experiment and simulation are based on the premise that the piston moving frequency is $18 \mathrm{~Hz}$ and the piston moving stroke is $20 \mathrm{~mm}$ to realize the alternate changes of volume. The initial stage when the volume begins to increase, the bottom of the container is constantly formed with cavitation bubbles, and the upper surface also has a large quantity of cavitation bubbles, which can be regarded as the stage of cavitation bubble formation. As the volume continues to increase, cavitation cloud separation phenomenon occurs, and other small bubbles are generated in the separation process, which can be regarded as the development stage of cavitation cloud. Finally, when the volume returns to its original size, the cavitation collapse stage occurs.

2. VAC is a new cavitation generation method, which needs to be further studied. The position of generating cavitation and the law of cavitation evolution are studied here. The subsequent studies can include piston motion frequency, piston motion stroke, different liquid phase and other aspects. 
3. The study of VAC has momentous research significance and paves the way for its application in industry in the future. For example: accurate polishing of complex microcavity, rapid manufacturing of emulsion, cavitation cleaning and so on.

\section{Declarations}

Availability of data and materials

The datasets used or analysed during the current study are available from the corresponding author on reasonable request.

Funding

National Nature Science Foundation of China (NSFC) (51575245), the Key Project of Jiangsu Province (BE2015134) Key Research and Development Project of Jiangsu Zhenjiang (KZ2020001).

Acknowledgements

The financial support provided by National Nature Science Foundation of China (NSFC) (51575245) and the Key Project of Jiangsu Province (BE2015134) is especially acknowledged and also grateful to Key Research and Development Project of Jiangsu Zhenjiang (KZ2020001).

Authorship contribution statement

Shangshuang Chen: Conceptualization, Methodology, Validation, Formal analysis, Investigation, Data curation, Resources, Writing - Original Draft, Visualization, Supervision, Project administration. Yun Wang: Conceptualization, Methodology, Formal analysis, Investigation, Resources, Writing - review \& editing, Funding acquisition. Fuzhu Li: Writing - review \& editing, Supervision, Project administration. Shenwei Xue: Formal analysis, Data curation, Writing - review \& editing, Supervision. Zhenying Xu: Writing - review \& editing, Supervision. Chao Yu: Writing - review \& editing, Supervision. Kun Zhang: Resources, Writing review \& editing, Supervision.

Declaration of interests

The authors declare that they have no known competing financial interests or personal relationships that could have appeared to influence the work reported in this paper.

\section{References}

1. Thanekar P, Gogate PR, Znak Z, Sukhatskiy Y, Mnykh R. Degradation of benzene present in wastewater using hydrodynamic cavitation in combination with air. Ultrason Sonochem. 2020;70:105296.

2. Sun X, Liu J, Ji L, Wang G, Zhao S, Yoon JY, et al. A review on hydrodynamic cavitation disinfection: The current state of knowledge. Sci Total Environ. 2020;737:139606. 
3. Sarc A, Stepisnik-Perdih T, Petkovsek M, Dular M. The issue of cavitation number value in studies of water treatment by hydrodynamic cavitation. Ultrason Sonochem. 2017;34:51-9.

4. Fujisawa N, Kikuchi T, Fujisawa K, Yamagata T. Time-resolved observations of pit formation and cloud behavior in cavitating jet. Wear. 2017;386-387:99-105.

5. Nagalingam AP, Thiruchelvam VC, Yeo SH. A novel hydrodynamic cavitation abrasive technique for internal surface finishing. Journal of Manufacturing Processes. 2019;46:44-58.

6. Petkovsek M, Dular M. Cavitation dynamics in water at elevated temperatures and in liquid nitrogen at an ultrasonic horn tip. Ultrason Sonochem. 2019;58:104652.

7. Tan KL, Yeo SH. Bubble dynamics and cavitation intensity in milli-scale channels under an ultrasonic horn. Ultrason Sonochem. 2019;58:104666.

8. Cui J, Zhang Z, Liu X, Liu L, Peng J. Analysis of the viscosity reduction of crude oil with nano-Ni catalyst by acoustic cavitation. Fuel. 2020;275.

9. Shen Z, Shao J, Zhang J, Qu W. Ultrasound cavitation enhanced chemotherapy: In vivo research and clinical application. Experimental Biology and Medicine. 2020;245(14):1200-12.

10. Priyadarshi A, Khavari M, Subroto T, Conte M, Prentice P, Pericleous K, et al. On the governing fragmentation mechanism of primary intermetallics by induced cavitation. Ultrason Sonochem. 2020;70:105260.

11. Zhang Y, Chen F, Zhang Y, Zhang Y, Du X. Experimental investigations of interactions between a laser-induced cavitation bubble and a spherical particle. Experimental Thermal and Fluid Science. 2018;98:645-61.

12. Zhang $Y$, Qiu X, Zhang $X$, Tang N, Zhang Y. Collapsing dynamics of a laser-induced cavitation bubble near the edge of a rigid wall. Ultrason Sonochem. 2020;67:105157.

13. Zhang $Y$, Xie X, Zhang Y, Zhang Y, Du X. Experimental study of influences of a particle on the collapsing dynamics of a laser-induced cavitation bubble near a solid wall. Experimental Thermal and Fluid Science. 2019;105:289-306.

14. Hemant J. Sagar, Moctar Oe. Dynamics ofa cavitation bubble near a solid surface and the induced damage. Journal of Fluids and Structures 2020;92.

15. Reuter F, Mettin R. Mechanisms of single bubble cleaning. Ultrason Sonochem. 2016;29:550-62.

16. Song Q-N, Xu N, Bao Y-F, Jiang Y-F, Gu W, Zheng Y-G, et al. Corrosion and Cavitation Erosion Behaviors of Two Marine Propeller Materials in Clean and Sulfide-Polluted 3.5\% NaCl Solutions. Acta Metallurgica Sinica (English Letters). 2017;30(8):712-20. 
17. Lu A, Shang Z, Luo X, Jin T, Luo H. Rapid fabrication of ultra-smooth Y-TZP bioceramic surfaces by dual-axis wheel polishing: process development and tribological characterization. Journal of Manufacturing Processes. 2020;55:276-87.

18. Babina K, Polyakova M, Sokhova I, Doroshina V, Arakelyan M, Novozhilova N. The Effect of Finishing and Polishing Sequences on The Surface Roughness of Three Different Nanocomposites and Composite/Enamel and Composite/Cementum Interfaces. Nanomaterials (Basel). 2020;10(7).

19. Zhou J, Niu X, Yang C, Huo Z, Lu Y, Wang Z, et al. Surface action mechanism and planarization effect of sarcosine as an auxiliary complexing agent in copper film chemical mechanical polishing. Applied Surface Science. 2020;529.

20. Wang G, Feng Z, Hu Y, Liu J, Zheng Q. Effects of Anisotropy on Single Crystal Silicon in Polishing Non-Continuous Surface. Micromachines (Basel). 2020;11(8).

21. Hutli E, Nedeljkovic MS, Bonyár A, Radovic NA, Llic V, Debeljkovic A. The ability of using the cavitation phenomenon as a tool to modify the surface characteristics in micro- and in nano-level. Tribology International. 2016;101:88-97.

22. Hutli E, Bonyár A, Oszetzky D, Nedeljkovic MS. Plastic deformation and modification of surface characteristics in nano- and micro-levels and enhancement of electric field of FCC materials using cavitation phenomenon. Mechanics of Materials. 2016;92:289-98.

23. Cheng L, Li J, Xue X, Tang B, Kou H, Perroud O, et al. Effect of $\beta / B 2$ phase on cavitation behavior during superplastic deformation of TiAl alloys. Journal of Alloys and Compounds. 2017;693:749-59.

24. Soyama H, Takeo F. Comparison between cavitation peening and shot peening for extending the fatigue life of a duralumin plate with a hole. Journal of Materials Processing Technology. 2016;227:80-7.

25. Alfonso-Muniozguren P, Hazzwan Bohari M, Sicilia A, Avignone-Rossa C, Bussemaker M, Saroj D, et al. Tertiary treatment of real abattoir wastewater using combined acoustic cavitation and ozonation. Ultrasonics Sonochemistry. 2020;64.

26. Sáez-Bastante J, Carmona-Cabello M, Pinzi S, Dorado MP. Recycling of kebab restoration grease for bioenergy production through acoustic cavitation. Renewable Energy. 2020;155:1147-55.

27. Panda D, Saharan VK, Manickam S. Controlled Hydrodynamic Cavitation: A Review of Recent Advances and Perspectives for Greener Processing. Processes. 2020;8(2).

28. Sun X, Xuan X, Song Y, Jia X, Ji L, Zhao S, et al. Experimental and numerical studies on the cavitation in an advanced rotational hydrodynamic cavitation reactor for water treatment. Ultrason Sonochem. 2021;70:105311. 
29. Kosel J, Sinkovec A, Dular M. A novel rotation generator of hydrodynamic cavitation for the fibrillation of long conifer fibers in paper production. Ultrason Sonochem. 2019;59:104721.

30. Kosel J, Sustarsic M, Petkovsek M, Zupanc M, Sezun M, Dular M. Application of (super)cavitation for the recycling of process waters in paper producing industry. Ultrason Sonochem. 2020;64:105002.

31. Chen F, Wang H, Yin S, Huang S, Tang Q, Luo H. Cavitation water-suction polishing of metallic materials under negative-pressure effect. Journal of Materials Processing Technology. 2019;273.

32. Chen F, Wang H, Tang Y, Yin S, Huang S, Zhang G. Novel cavitation fluid jet polishing process based on negative pressure effects. Ultrason Sonochem. 2018;42:339-46.

33. Kauer M, Belova-Magri V, Cairos C, Schreier HJ, Mettin R. Visualization and optimization of cavitation activity at a solid surface in high frequency ultrasound fields. Ultrason Sonochem. 2017;34:474-83.

34. Li F. Study on Dynamic Evolution of Cavitation Clouds and Optimization of Standoff Distance in Water Cavitation Peening. Journal of Mechanical Engineering. 2019;55(9).

35. Cai SQ. Cavitation occurring in capillary tubes. Physics Letters A. 2019;383(6):509-13.

36. Chen TN, Guo ZN, Zeng BW, Yin SH, Deng Y, Li HH. Experimental research and numerical simulation of the punch forming of aluminum foil based on a laser-induced cavitation bubble. The International Journal of Advanced Manufacturing Technology. 2017;93(9-12):3275-84.

\section{Figures}




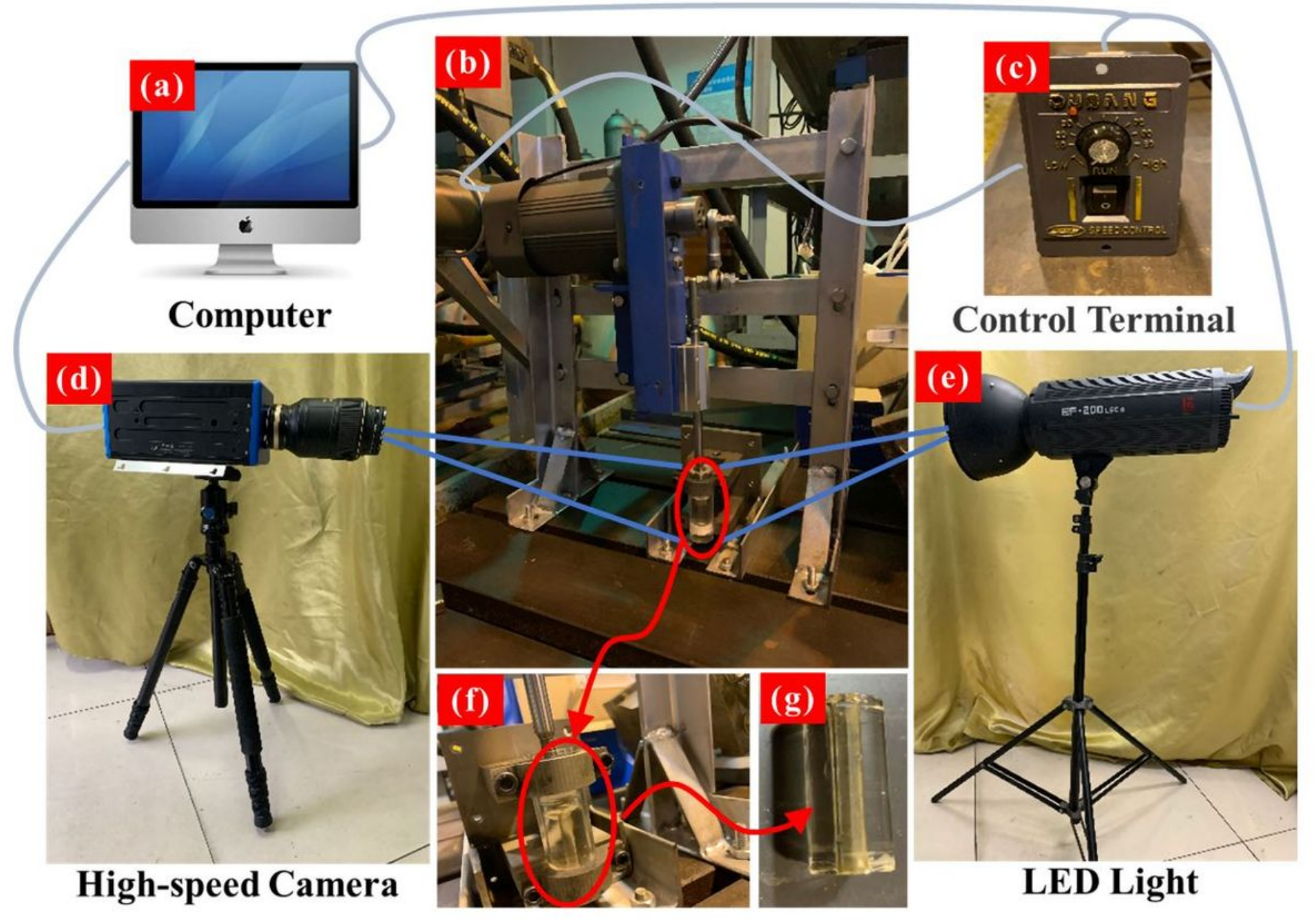

Figure 1

Experimental platform: (a) computer, (b) motor drive device, (c) control terminal, (d) high-speed camera, (e)LED light, (f) cavitation generator (g) sample 


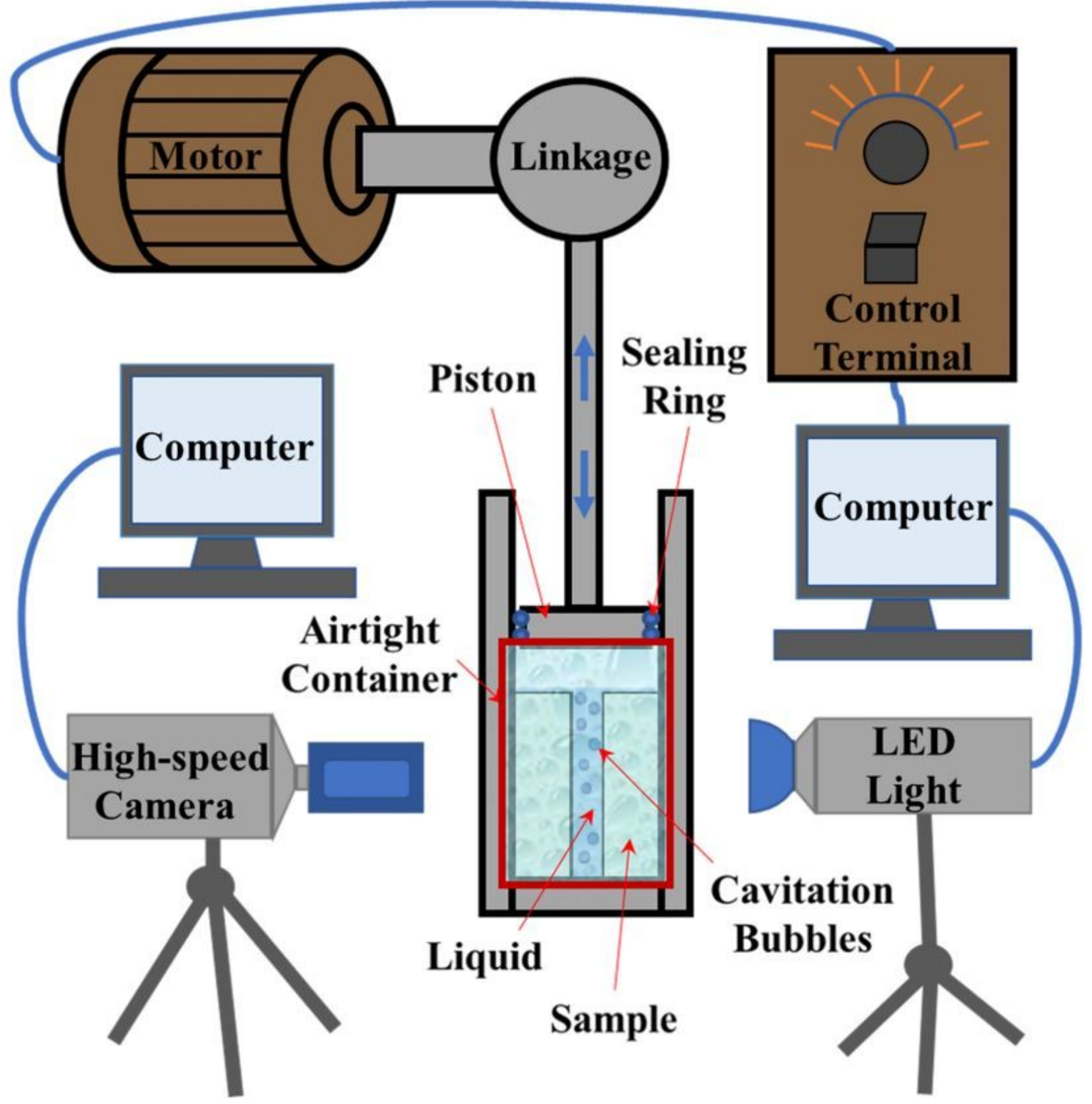

Figure 2

Schematic diagram of the VAC 


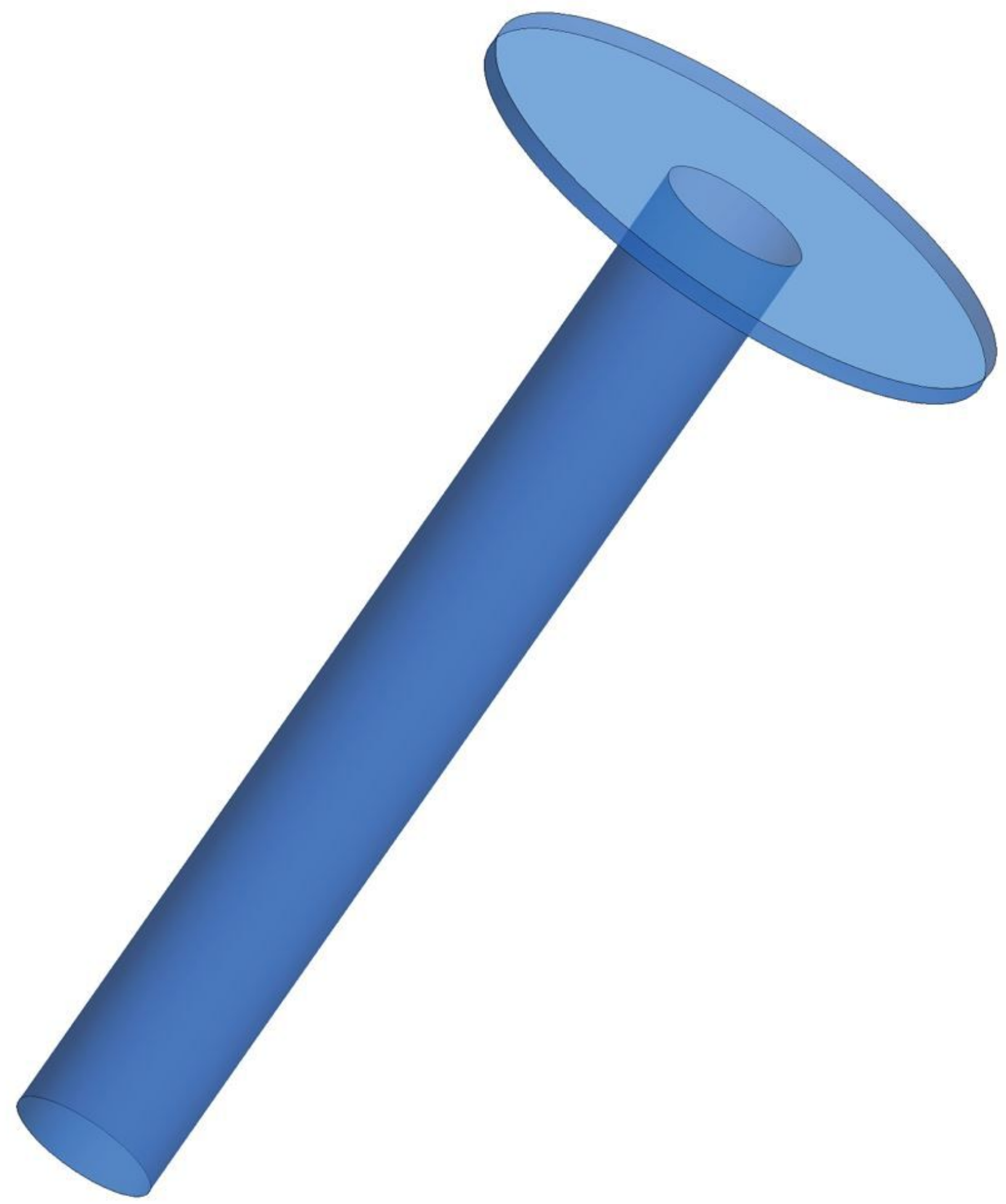

Figure 3

Simulation model 


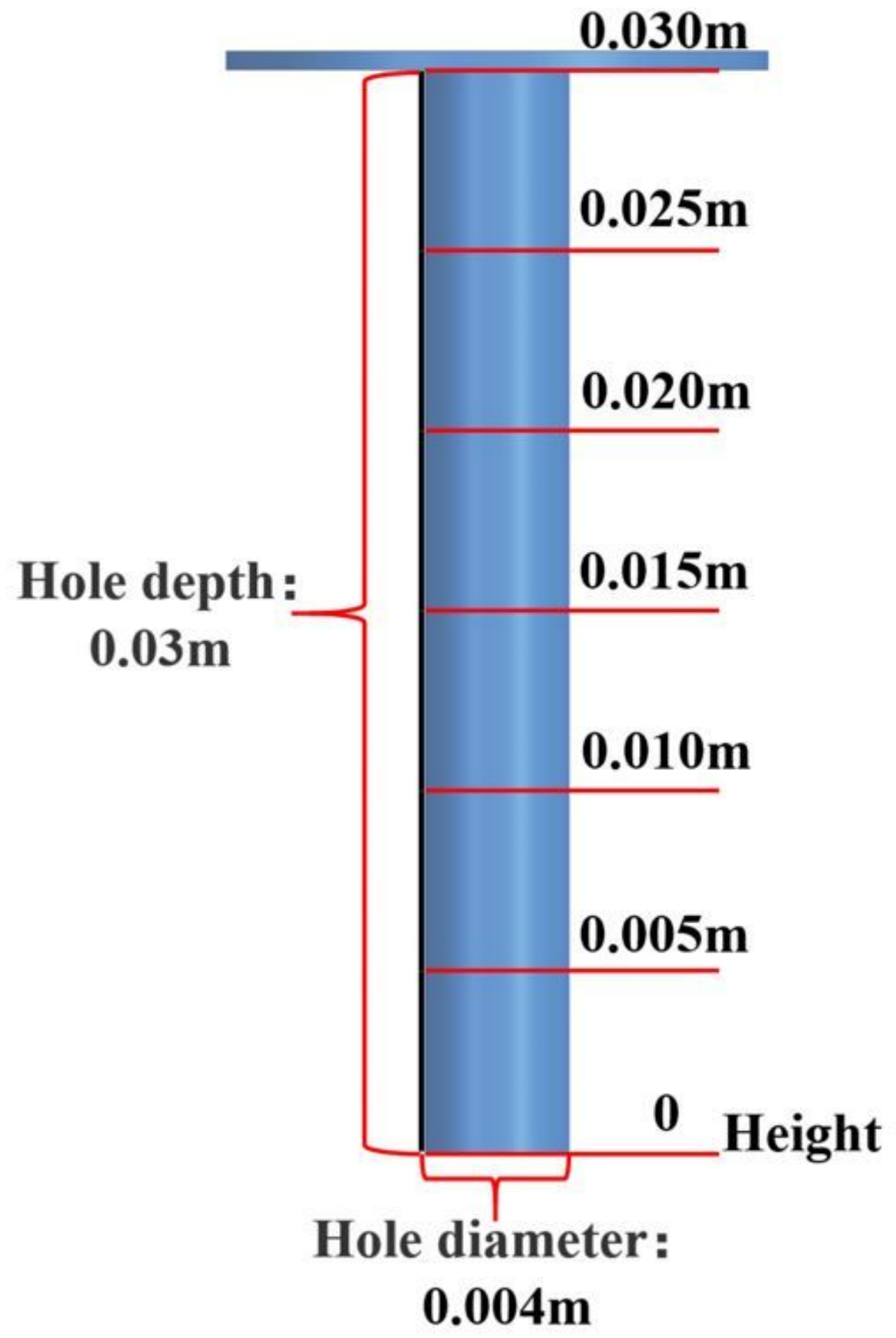

Figure 4

Model size diagram 

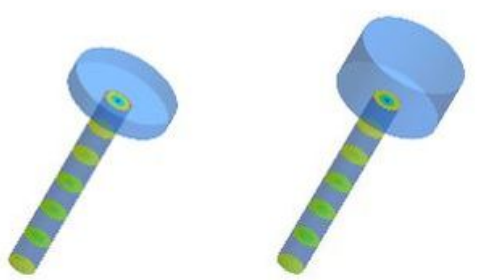

$5.56 \mathrm{~ms}$

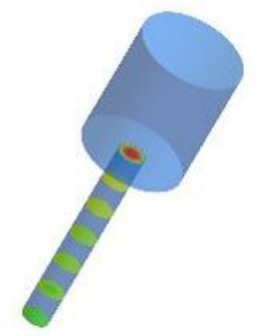

n.

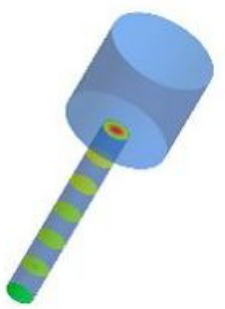

$\cdots \cdots$

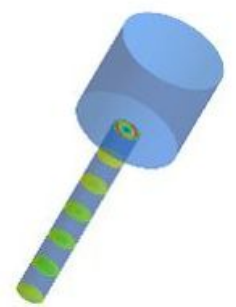

$16.67 \mathrm{~ms}$

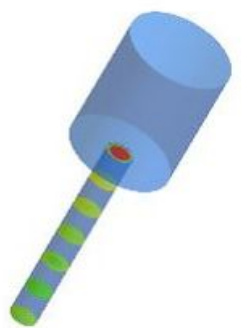

$22.22 \mathrm{~ms}$
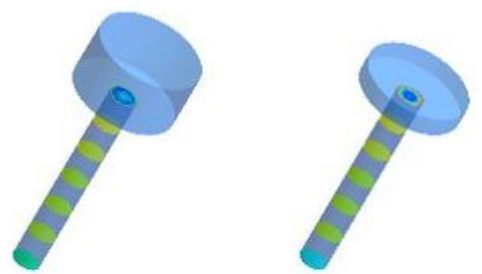

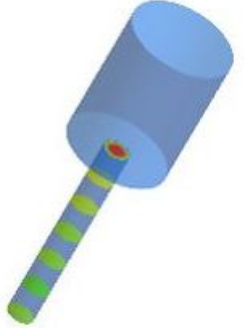

$27.78 \mathrm{~ms}$

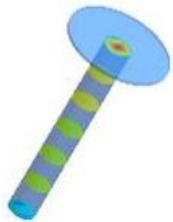

$\cdots n$
Vapor.Volume Fraction Contour 7

$9.895 \mathrm{e}-001$

9.377e-001

$8.858 \mathrm{e}-001$

$8.339 \mathrm{e}-001$

$7.821 \mathrm{e}-001$

$7.302 \mathrm{e}-001$

$6.784 \mathrm{e}-001$

$6.265 \mathrm{e}-001$

$5.746 \mathrm{e}-001$

$5.228 \mathrm{e}-001$

$4.709 \mathrm{e}-001$

$4.191 \mathrm{e}-001$

3.672e-001

$3.153 e-001$

$2.635 \mathrm{e}-001$

$2.116 \mathrm{e}-001$

$1.598 \mathrm{e}-001$

$1.079 \mathrm{e}-001$

$5.605 \mathrm{e}-002$

$4.194 \mathrm{e}-003$

Figure 5

Periodic cloud map 


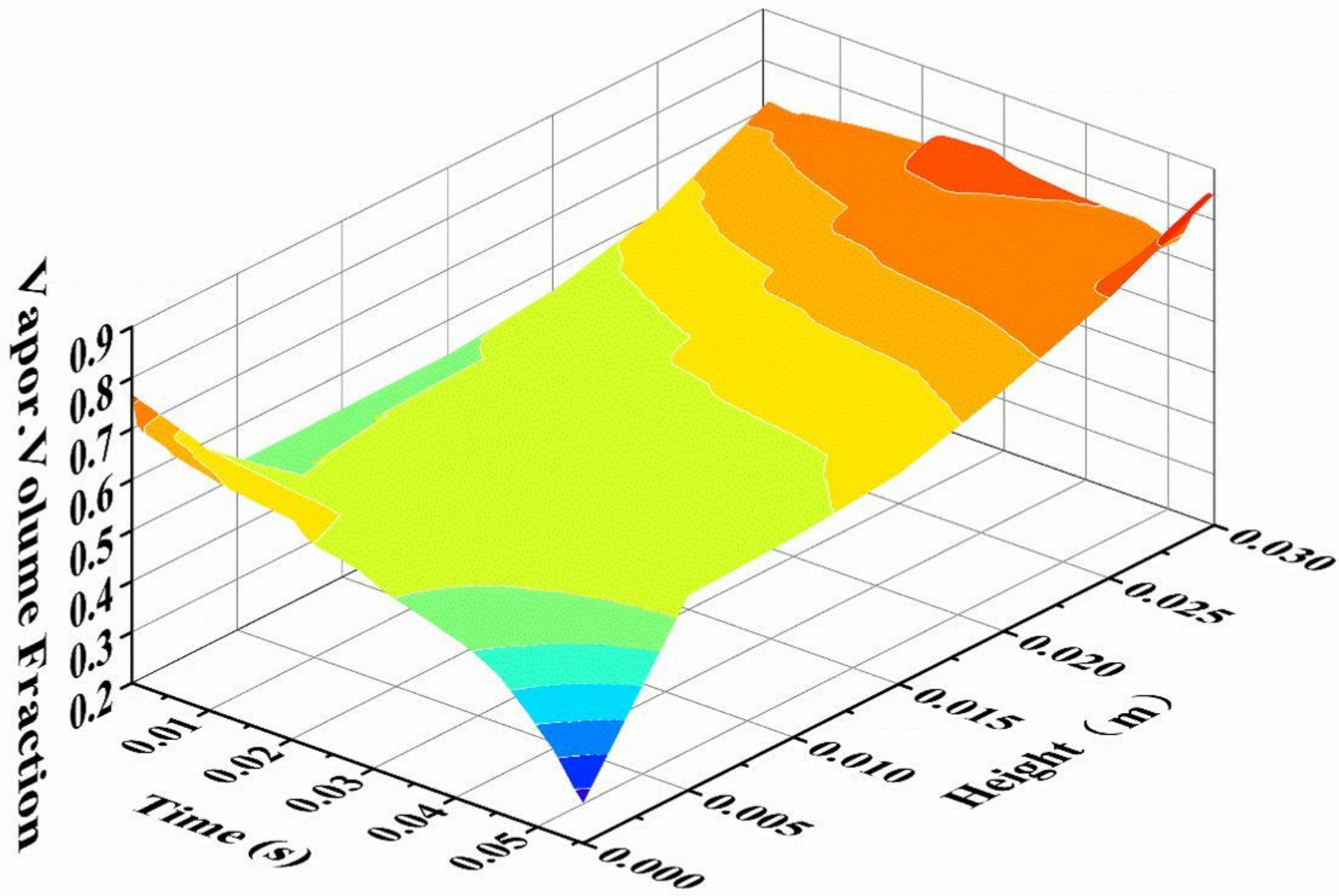

Figure 6

Gas phase volume distribution at different heights along the central axis of the inner hole with time 


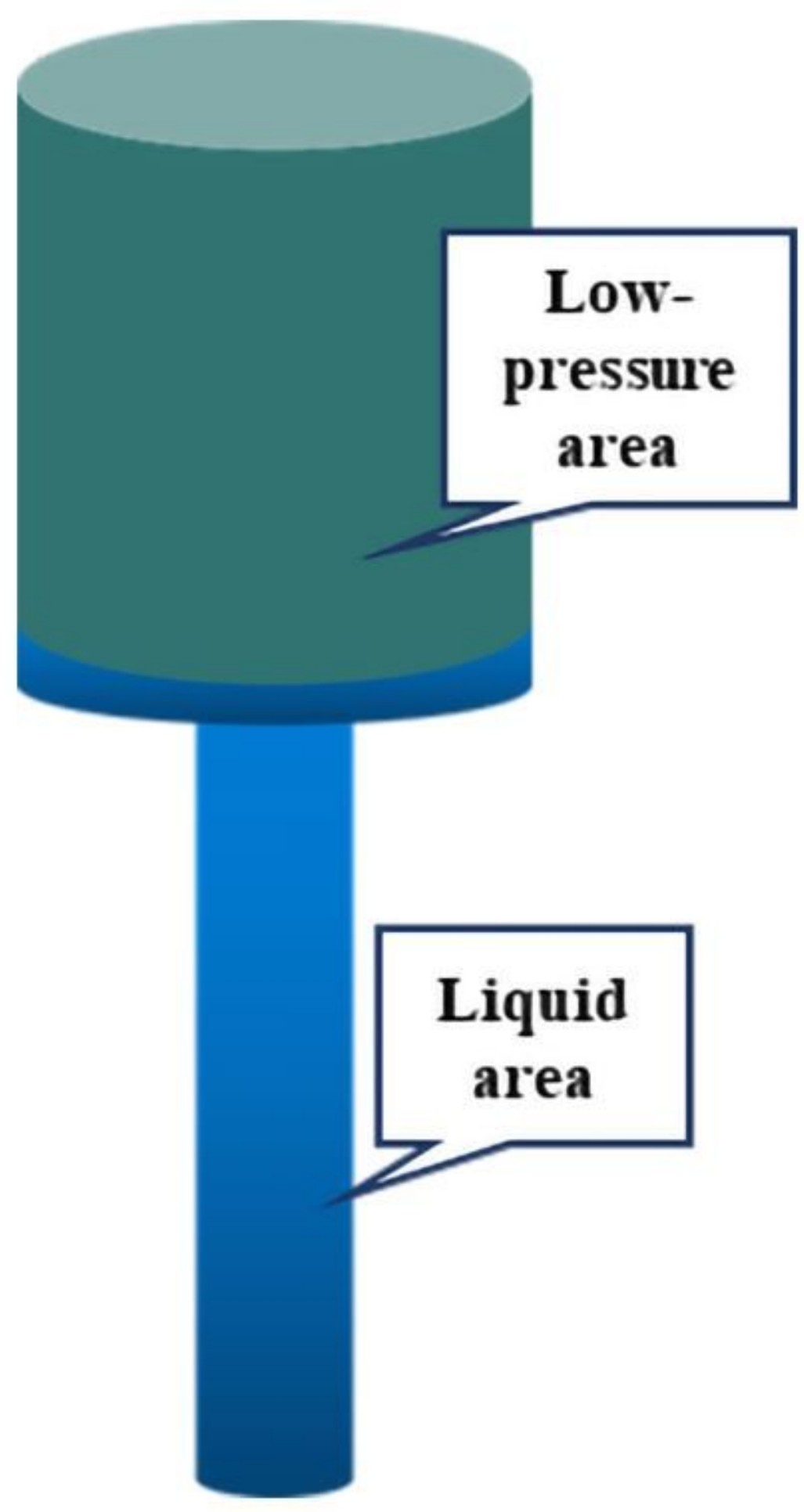

Figure 7

Schematic diagram of low-pressure zone in vacuum-like environment 


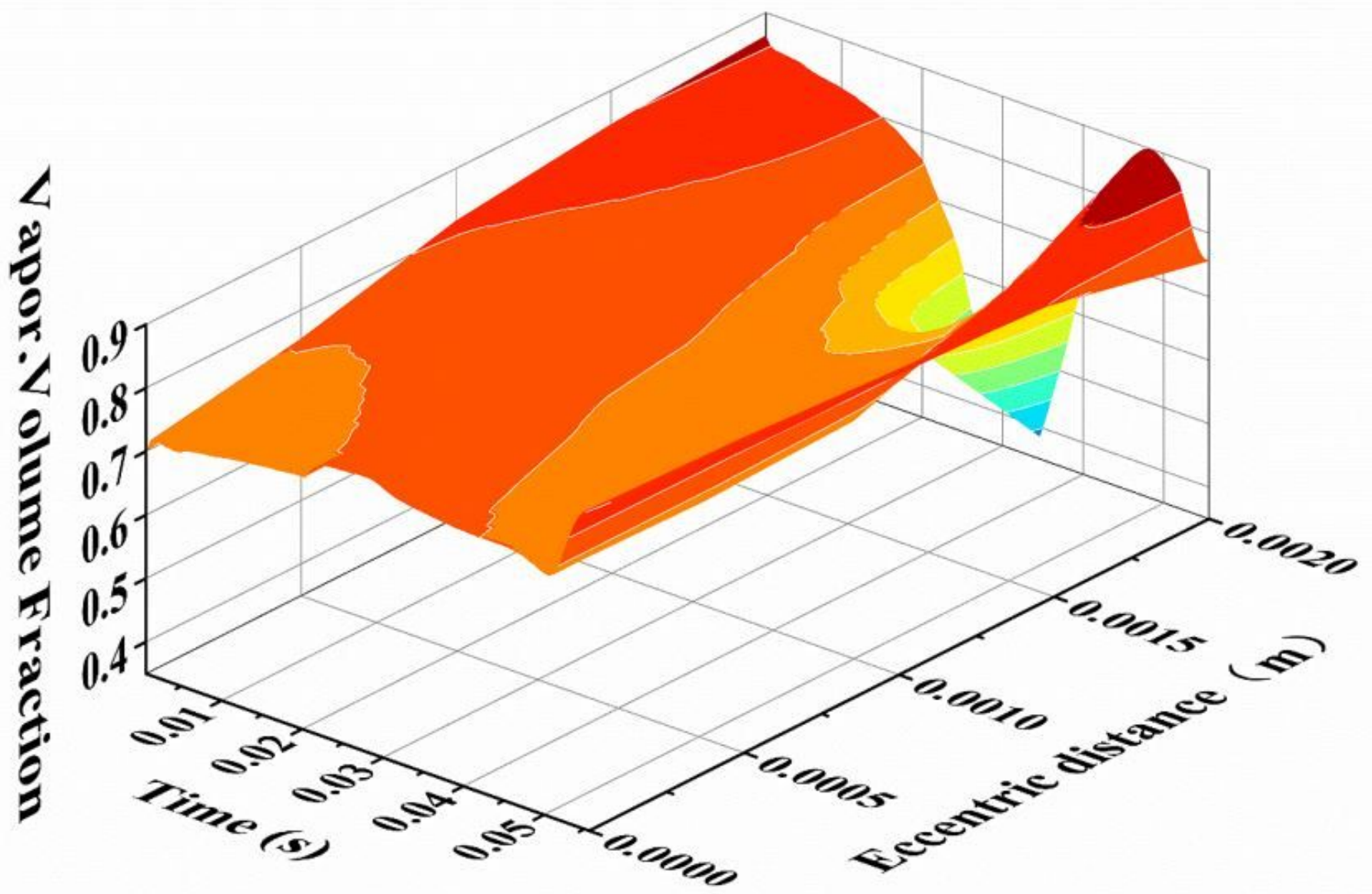

Figure 8

Variation of gas phase volume fraction with different eccentricity on the upper surface over time 


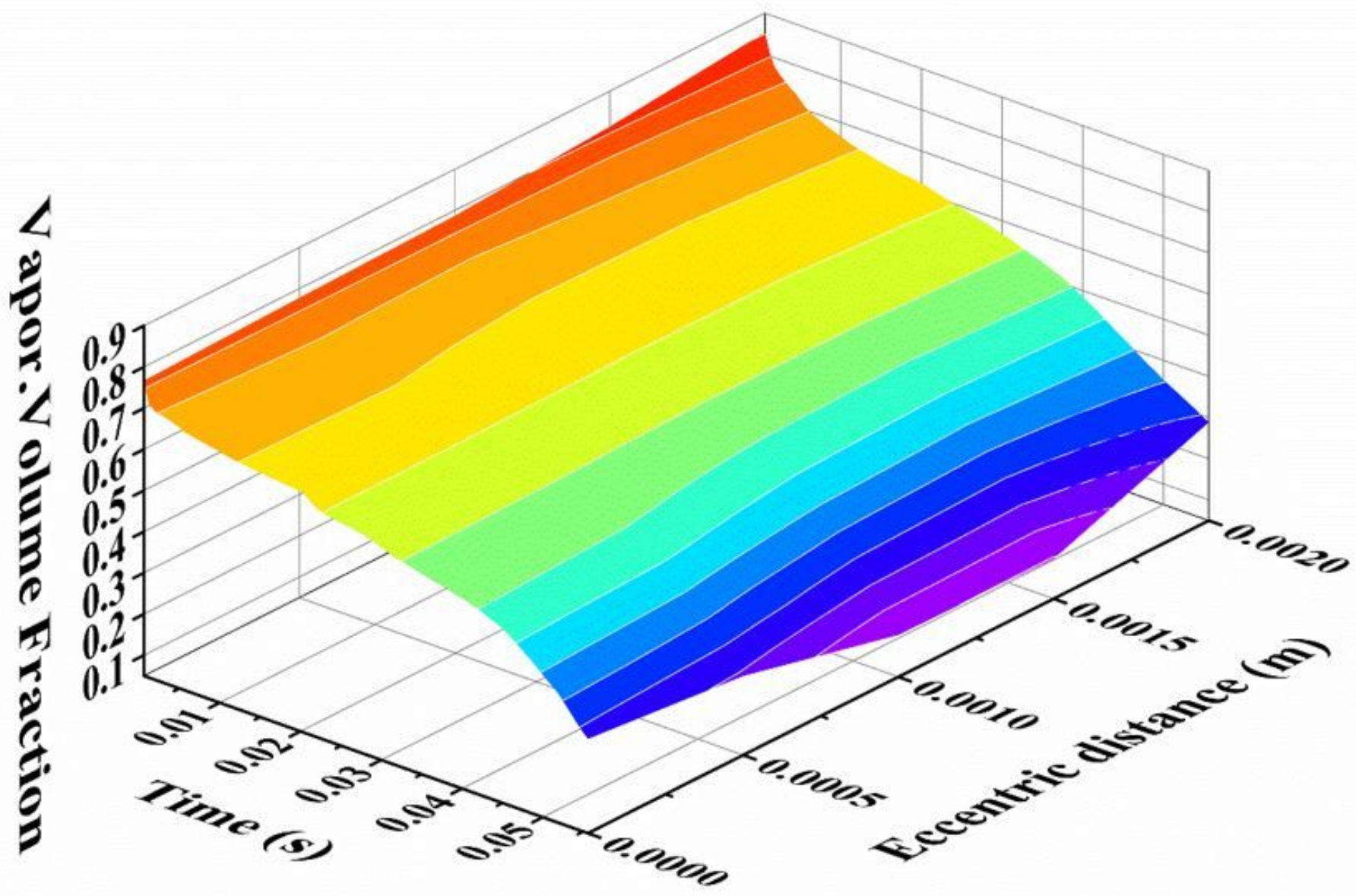

Figure 9

Variation of gas phase volume fraction with different eccentricity at the bottom surface over time 


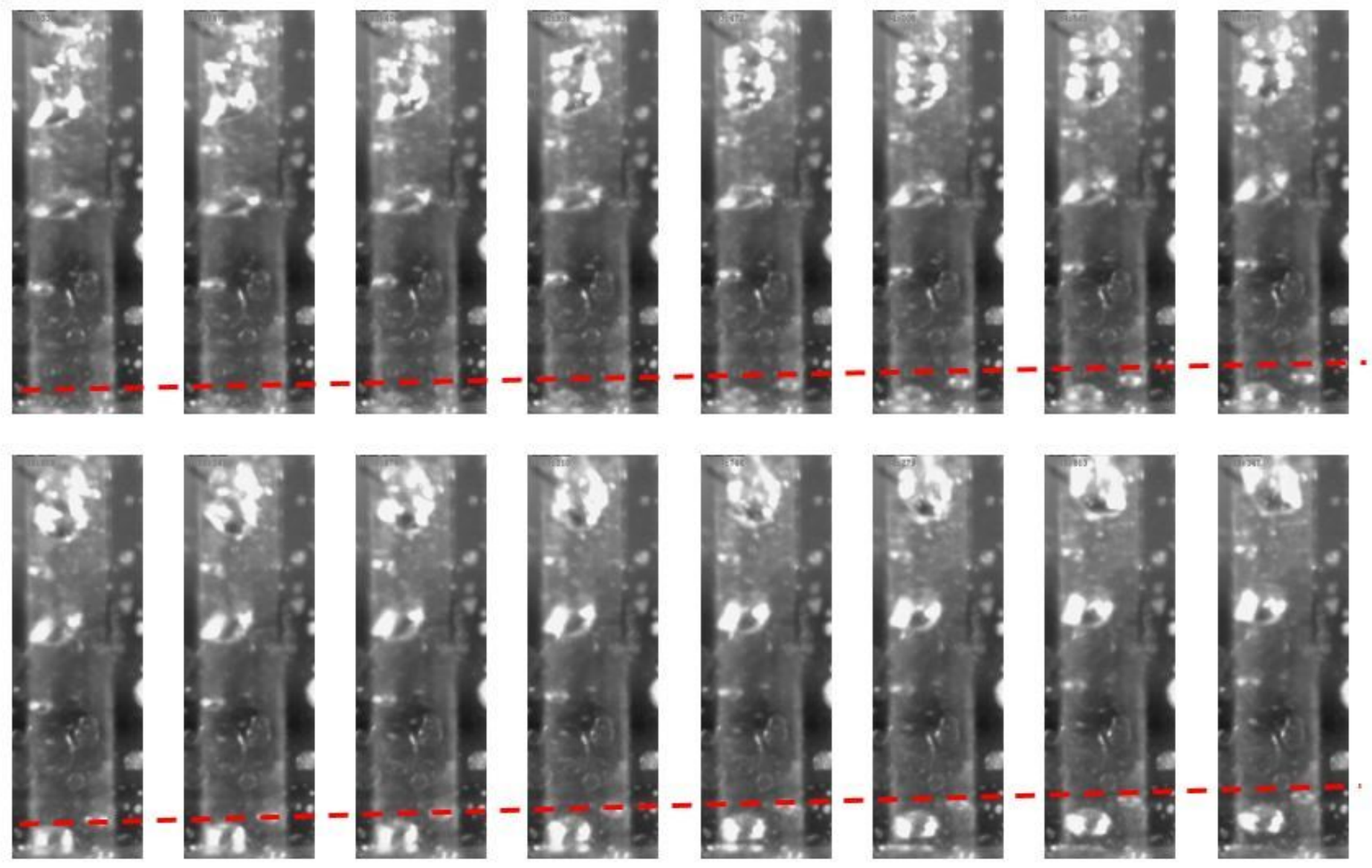

Figure 10

Diagram of cavitation bubble formation

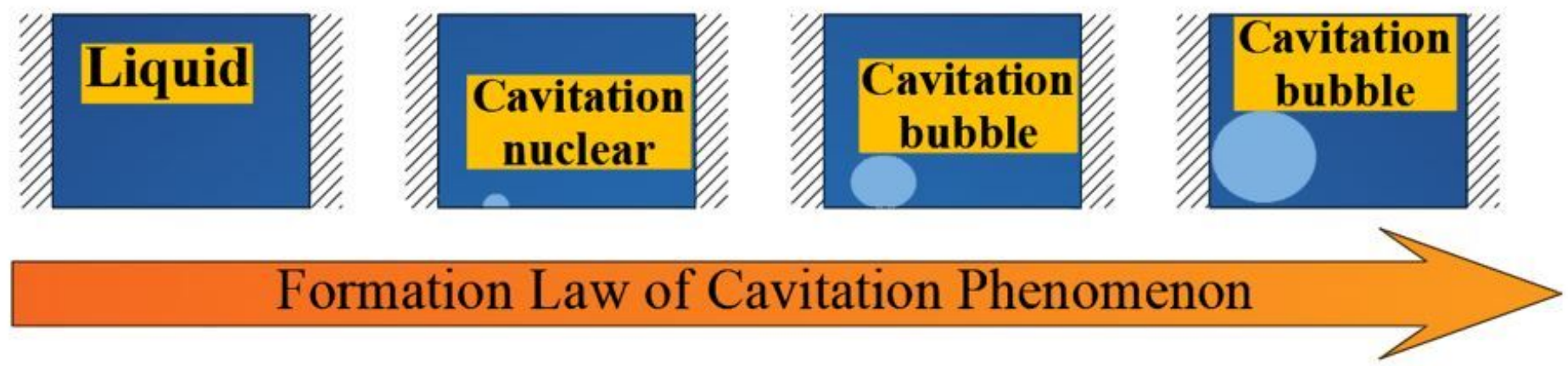

Figure 11 


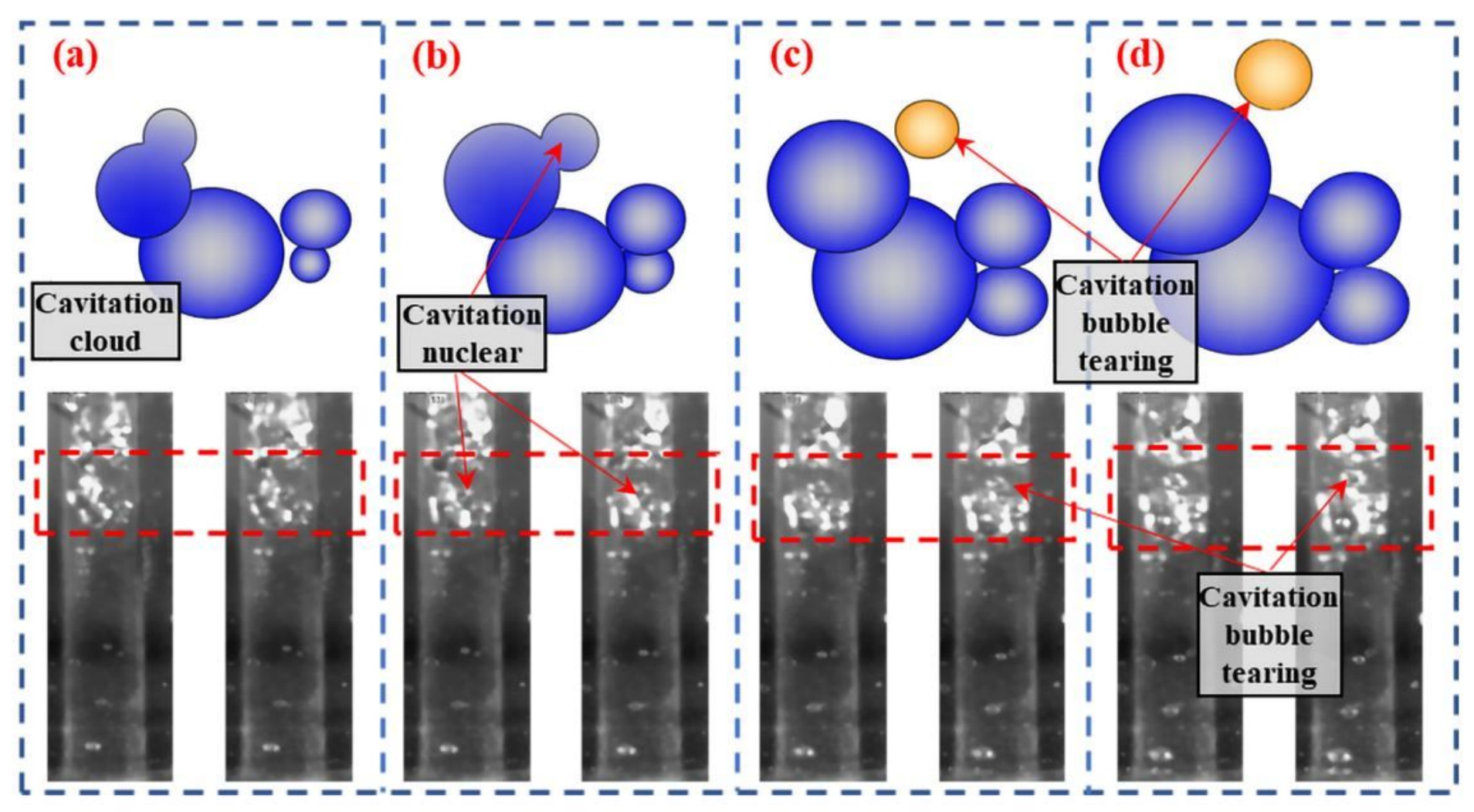

Figure 12

Diagram of cavitation bubbles development 
(a)

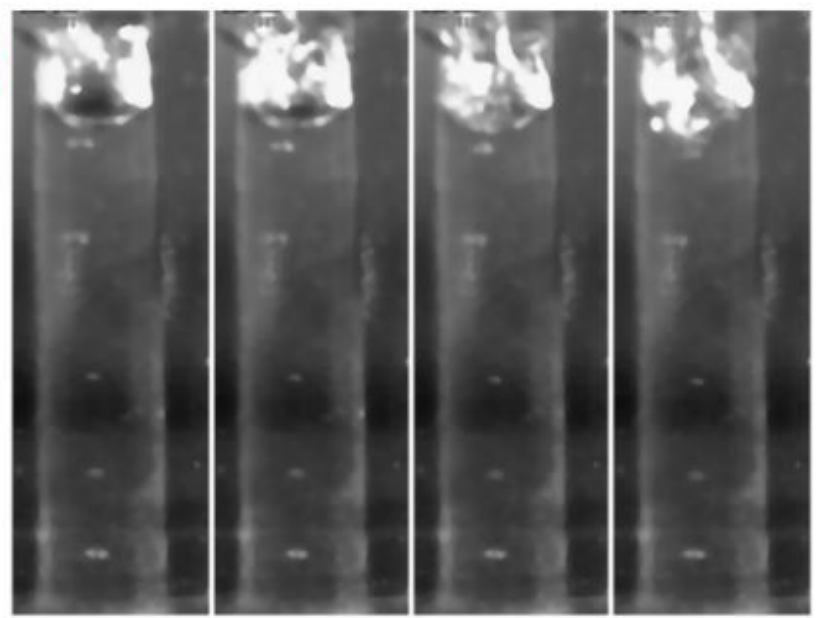

(c)

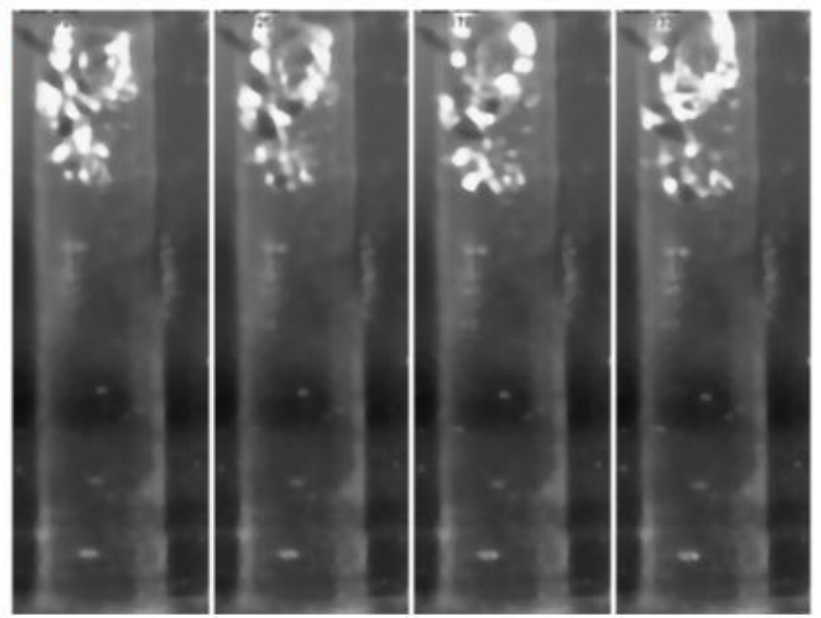

(b)

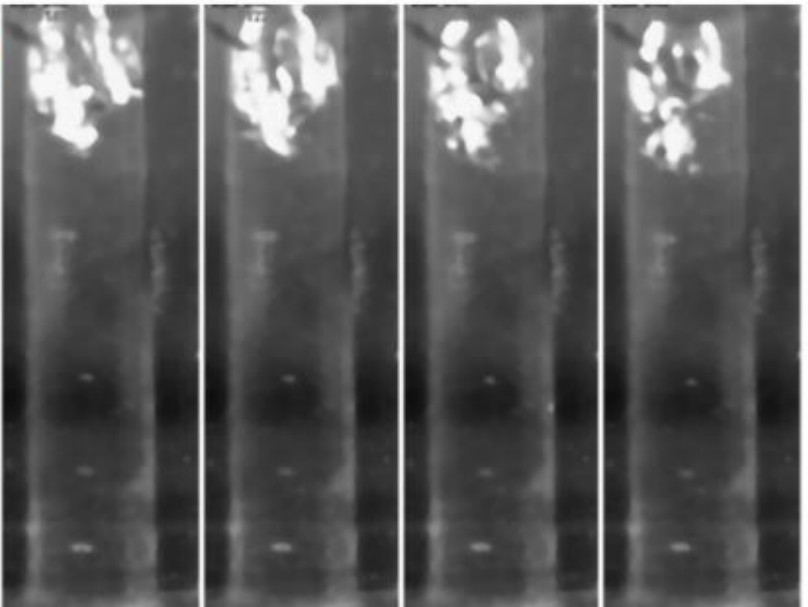

(d)

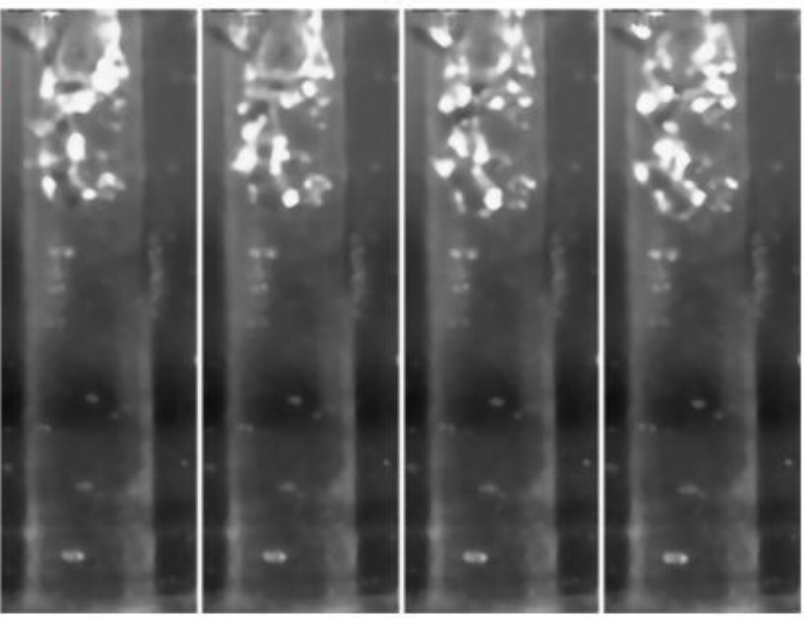

Figure 13

Diagram of cavitation bubbles collapse

(a)

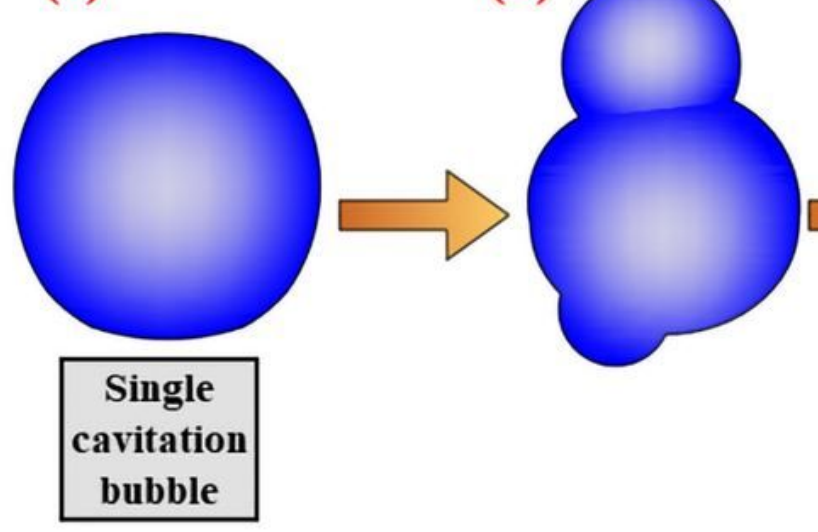

(c)

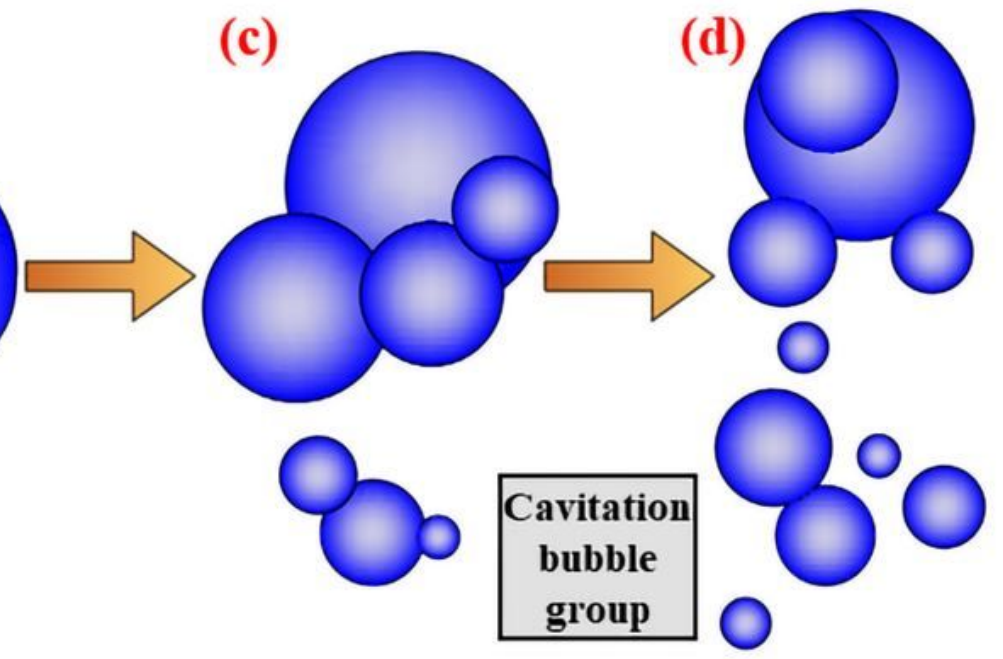


Figure 14

Schematic diagram of cavitation bubble collapse 